\title{
A Phase-changing Bistable Electroactive Polymer Exhibiting Sharp Rigid-to-Rubbery Transition
}

\author{
Zhi Ren, Wei Hu, Chao Liu, Shenshen Li, Xiaofan Niu, Qibing Pei * \\ Department of Materials Science and Engineering, University of California, Los Angeles, CA, \\ 90095, United States
}

\section{Supporting Information}

\section{Formation of the crystalline aggregates of stearyl moieties in the SA-UDA copolymers}

Wide-angle XRD experiments of BS60 were conducted at both room temperature and $70{ }^{\circ} \mathrm{C}$. At room temperature, the XRD signal comprises both contributions from amorphous segments of UDA moieties and crystalline aggregates of stearyl moieties. At $70{ }^{\circ} \mathrm{C}$, the phase transition, melting of crystalline aggregates of stearyl moieties, is completed; therefore, the XRD pattern consists of only the contribution from amorphous segments (wide peak indicating short range order only). The sharp XRD peak centered at $21^{\circ}$ at ambient temperature and its disappearance at $70{ }^{\circ} \mathrm{C}$ correspond to the reversible formation of the crystalline aggregates of the stearyl moieties in the SA-UDA copolymer. 


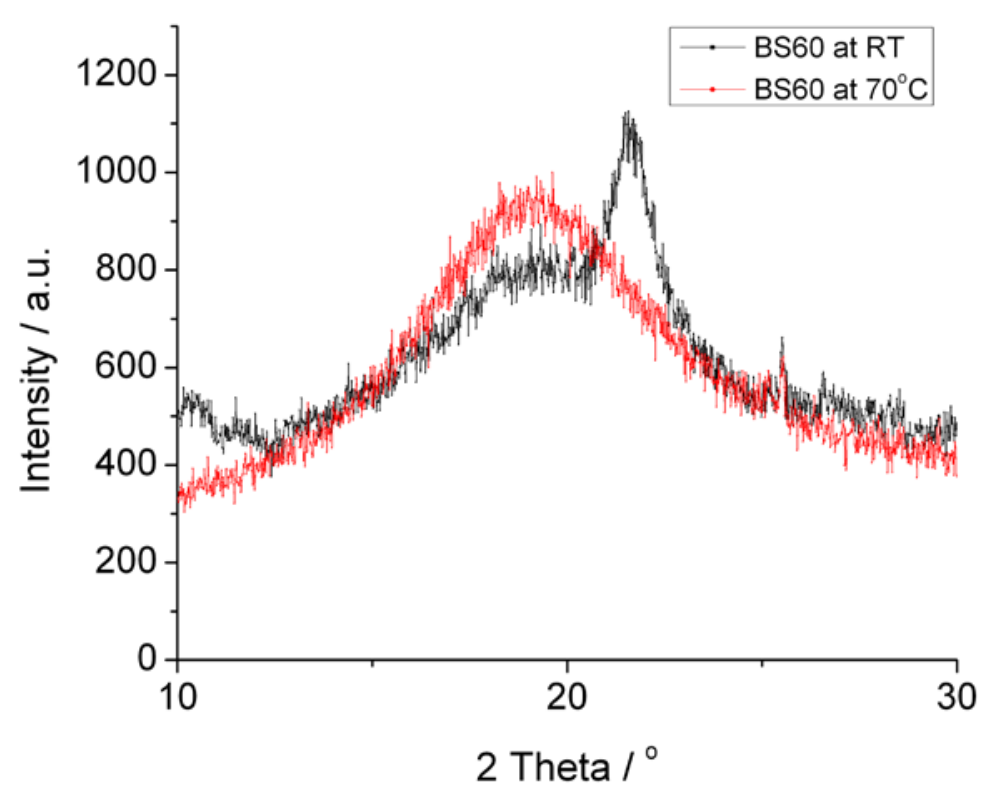

Figure SI-1. XRD of BS60 at room temperature and at $70{ }^{\circ} \mathrm{C}$

\section{Consistent melting temperature despite of different thermal history of BS60}

BS60 samples were fabricated and annealed at $60{ }^{\circ} \mathrm{C}$ for $2 \mathrm{hrs}$ before being characterized with DSC. An initial heating process (Fig. SI-2 Initial Heating) was conducted with a heating rate of 5 ${ }^{\circ} \mathrm{C} / \mathrm{min}$, showing an endothermic peak due to melting of stearyl acrylate moieties. Three consecutive cooling \& heating cycles were conducted afterwards, with different cooling rate (2 ${ }^{\circ} \mathrm{C} / \mathrm{min}$ for "Fig. SI-2 Cool-2-Heat-5", $5{ }^{\circ} \mathrm{C} / \mathrm{min}$ for "Fig. SI-2 Cool-5-Heat-5" and $10{ }^{\circ} \mathrm{C} / \mathrm{min}$ for “Fig. SI-2 Cool-10-Heat-5”, respectively) but identical heating rates $\left(5{ }^{\circ} \mathrm{C} / \mathrm{min}\right)$. With the increasing cooling rate, the samples showed a decreasing crystallization temperature. The heat flow (mW) integrated with time showed similar amount of heat (Joule) released during the crystallization process despite of different cooling rate. Furthermore, in spite of the different cooling rate or thermal history, the subsequent melting overlapped very well on the DSC diagrams. This result suggests that the phase transition (melting) temperature of BS60 is not affected by its thermal history, which is desirable in practical applications. 


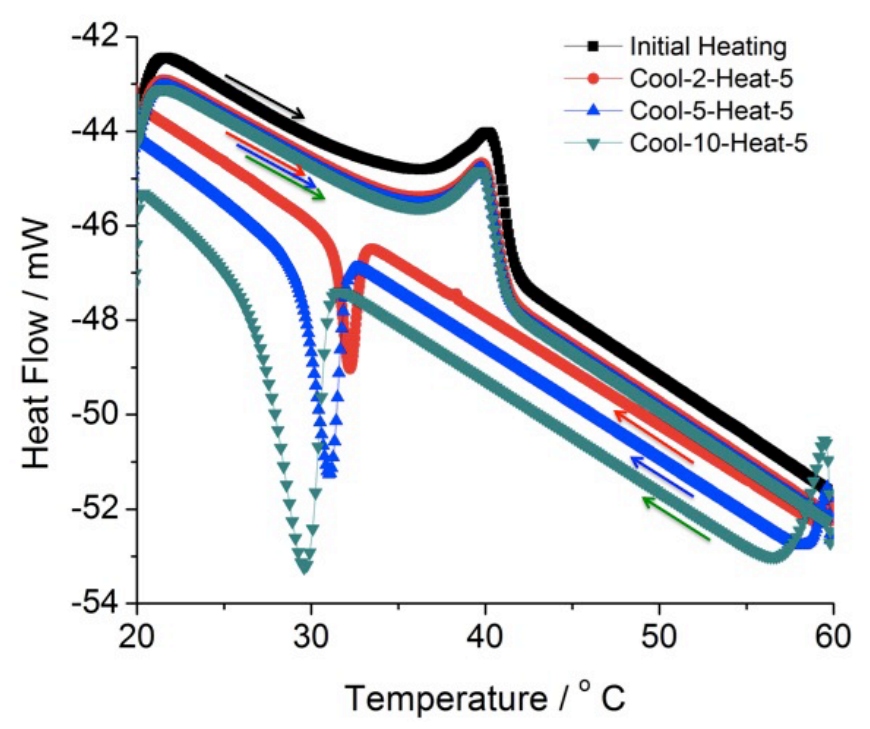

Figure SI-2. DSC characterization of BS60 with different cooling rate $\left(2^{\circ} \mathrm{C} / \mathrm{min}, 5{ }^{\circ} \mathrm{C} / \mathrm{min}, 10\right.$ $\left.{ }^{\circ} \mathrm{C} / \mathrm{min}\right)$ and identical heating rate $\left(5^{\circ} \mathrm{C} / \mathrm{min}\right)$. Arrows indicate temperature ramping directions. Curves labeled as "Cool-x-Heat-5" with cooling rate of $\mathrm{x}{ }^{\circ} \mathrm{C} / \mathrm{min}$ and heating rate of $5{ }^{\circ} \mathrm{C} / \mathrm{min}$.

\section{Upturns of modulus in the stress-strain curves}

There are generally two suspicious causes for an upturn appearing in a stress-strain curve. One is strain-induced crystallization; the other is limited-chain extensibility. With lowering the SA content in the SA-UDA copolymers, the upturn appears at even earlier strain, indicative of little contribution from strain-induced crystallization of SA moieties to the upturns. Samples of 0SA/100UDA exhibit similar upturn of modulus at room temperature and at $50{ }^{\circ} \mathrm{C}$. The independence of temperature on the upturning strain of 0SA/100UDA suggests that the crystallization of the UDA moieties during elongation can also be ruled out. Therefore, the upturning effect is mainly attributed to the limited-chain extensibility of the polymer chains. Copolymerization of small molecular monomer (SA) and trifunctional crosslinker (TMP-TA) with UDA having long flexible chains results in a network with varying chain length in between 
crosslinks, which is similar to a bimodal structure. The flexible long chains of UDA retard early rupture of the SA-UDA copolymer. The relatively short chains comprising SA and TMP-TA provide limited chain extensibility, which results in rapid upturns. The upturning strain defined in the Fig. 5 inset (BS60 as an example) is decreased as lowering SA:UDA ratio from BS70 to BS40. A plausible reason might be that by lowering the SA content, the short chains become shorter and the density of short chains also increases as the crosslink density increases, resulting in upturns in smaller strains. Samples of 0SA/100UDA-0TMP-TA exhibit upturn at a much larger strain than SA-UDA copolymers comprising small molecular components, further proving the important role of short chains in upturning of modulus as the polymer is stretched.

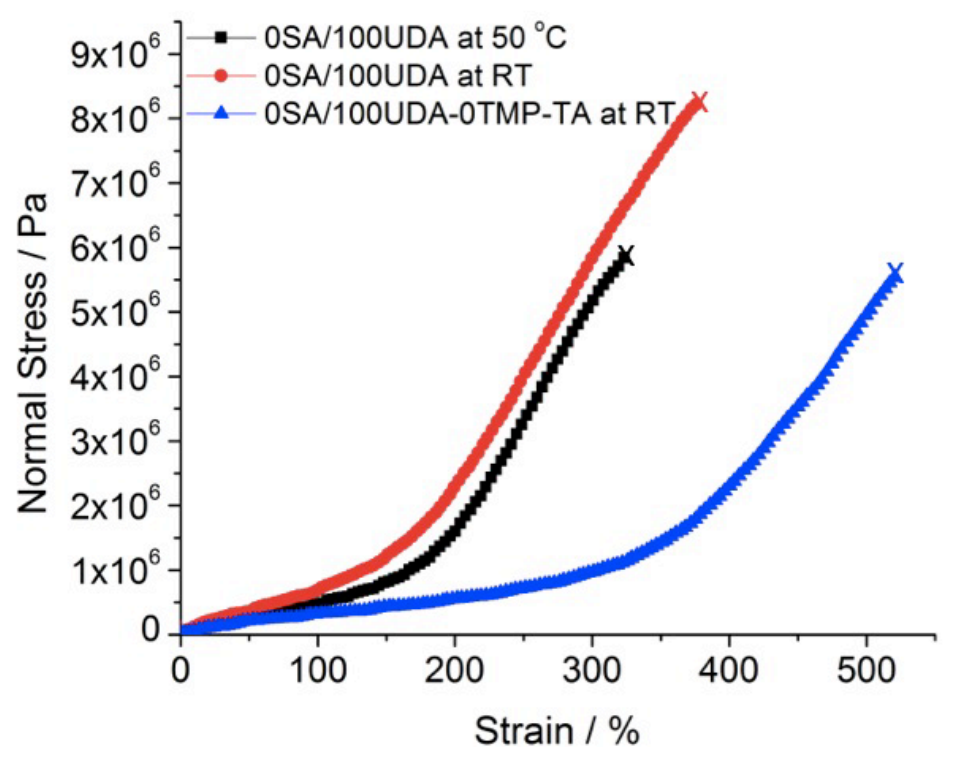

Figure SI-3. Stress-strain curve of $0 \mathrm{SA} / 100 \mathrm{UDA}$ at $50{ }^{\circ} \mathrm{C}$ and at room temperature, and 0SA/100UDA-0TMP-TA at room temperature. Marker $\mathrm{x}$ at the end of each curve indicates rupture. See Table SI-1 for the specific formulations of the each polymer.

The effects of TMP-TA and photoinitiators on the mechanical properties of BS60 
In addition to the ratio of SA:UDA, the small molecular crosslinker, TMP-TA, and photoinitiator (mixture of DMPA and Benzophenone at a ratio of 2:1 in weight) are also important formulation parameters that can influence the mechanical properties, phase transition temperature, and shape memory properties of the SA-UDA polymers. To understand the roles of TMP-TA and photoinitiator, BS60 was selected as the baseline material. With an increase of TMP-TA content from 1 to 2 parts (BS60 to BS60-2TMP-TA), the rigid-to-rubbery transition temperature decreases slightly, as shown in Fig. SI-4a. This trend is similar when the UDA content is increased in the SA-UDA copolymers. The storage modulus increases with the content of TMP-TA both in the rigid state and in the rubbery state (see Fig. SI-4b), due to higher crosslink density. The increasing TMP-TA content also lowers the elongation of the resulting copolymer, e.g., BS60 has an elongation of 250\% while it is only 150\% for BS60-1.5TMP-TA. For sufficient tensile strain while providing effective self-stiffening of the rubbery SA-UDA at large strains, 1 part TMP-TA was used for much of the present work described in the main body of the manuscript.
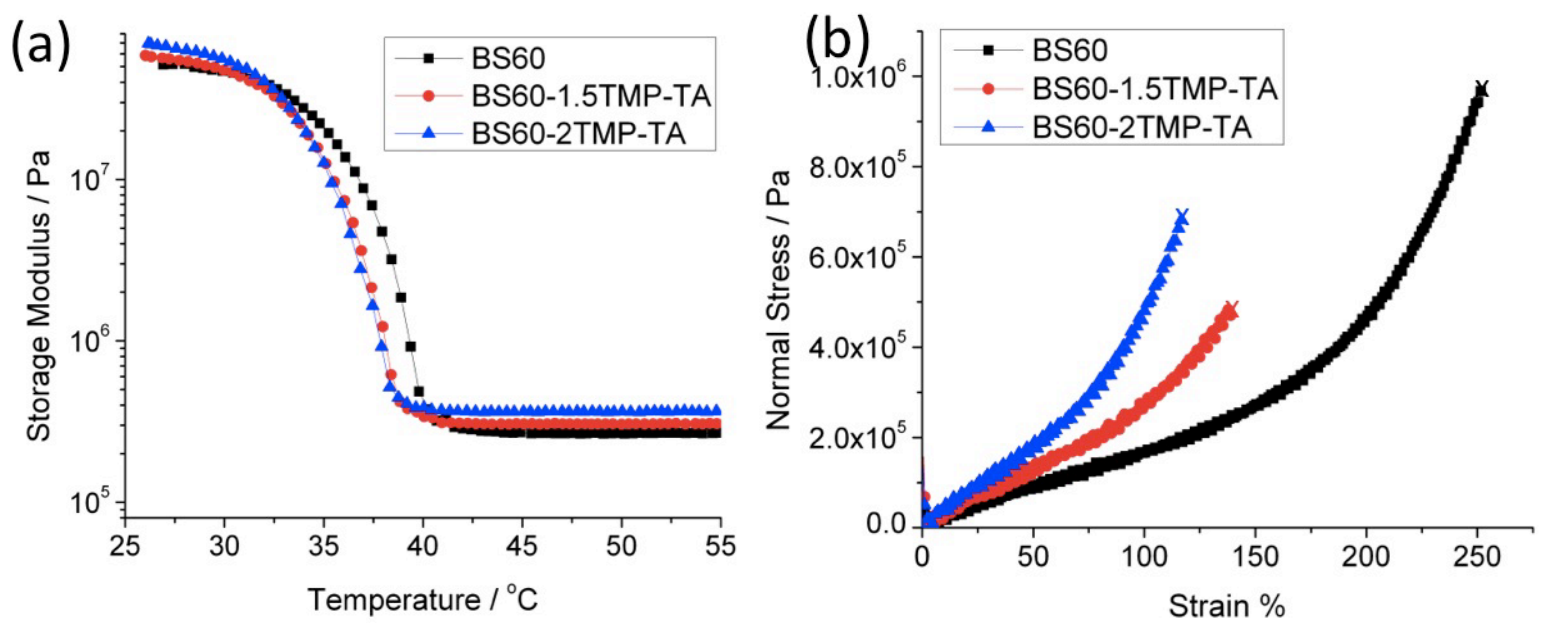

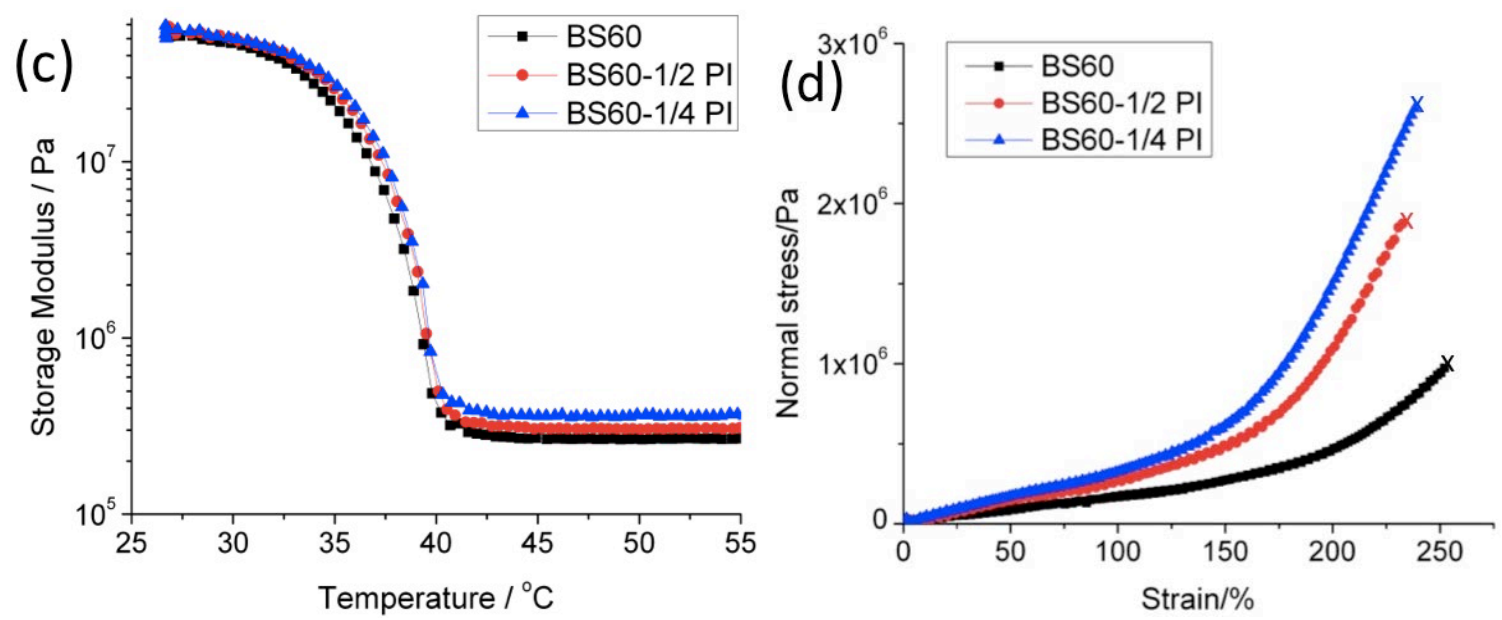

Figure SI-4. Evolution of storage modulus $(\mathrm{a}, \mathrm{c})$ as a function of temperature determined by DMA with temperature ramping from 25 to $55{ }^{\circ} \mathrm{C}$ at $2{ }^{\circ} \mathrm{C} \min ^{-1}$. Stress-strain curves of the polymers at $50{ }^{\circ} \mathrm{C}(\mathrm{b}, \mathrm{d})$. Marker $\mathrm{x}$ at the end of each curve indicates rupture. See Table SI-2 for the specific formulations of each polymer.

Mechanical properties of BS60 with varying amount of photoinitiators were also characterized (Fig SI-4c, d). The temperature dependent moduli of BS60, 'BS60-1/2 PI' and 'BS60-1/4 PI' were shown in Fig. SI-4c. The modulus does not change with the varying amount of photoinitiator at room temperature. The transition temperature range becomes slightly narrower with lower photoinitiator content. The moduli of all three materials plateau at temperatures above the transition, indicative of effective crosslinking even when the photoinitiator content is reduced by $75 \%$. The steady modulus at the rubbery state increases with lower photoinitiator content. This could be explained by the decreased number of dangling chain ends since, in principle, each photoinitiator introduces two chain ends. The increasing modulus is also shown in the normal stress-strain curves (Fig. SI-4d), obtained in the rubbery state. Compared with BS60 that possessed a tear-strength of $1 \mathrm{MPa}$, the 'BS60-1/4-PI' had a tear strength of 2.6MPa indicating that it is a tougher material. On the other hand, low photoinitiator content could lead to 
insufficient curing if the UV curing conditions were not strictly controlled, and the resulting polymer film would be difficult to peel off of the substrate. For convenience and reproducibility, we used 1part DMPA and 0.5part benzophenone for much of the present work described in the main body of the manuscript.

\section{Formulas of prepolymer solutions employed in Supporting Information}

Table SI-1. Formulas of prepolymer solutions employed to illustrate the upturn phenomenon (parts by weight, 50SA/50UDA is identical to BS50)

\begin{tabular}{|l|l|l|l|l|l|}
\hline Sample & SA & UDA & TMP-TA & DMPA & Benzophenone \\
\hline 50SA/50UDA & 50 & 50 & 1 & 1 & 0.5 \\
\hline 0SA/100UDA & 0 & 100 & 1 & 1 & 0.5 \\
\hline 100SA/0UDA & 100 & 0 & 1 & 1 & 0.5 \\
\hline 0SA/100UDA-0TMP-TA & 0 & 100 & 0 & 1 & 0.5 \\
\hline
\end{tabular}

Table SI-2. Formulas of prepolymer solutions employed to investigate the effects of TMA-TA and photoinitiators on the mechanical properties of BS60 (parts by weight)

\begin{tabular}{|l|l|l|l|l|l|}
\hline Sample & SA & UDA & TMP-TA & DMPA & Benzophenone \\
\hline BS60 & 60 & 40 & 1 & 1 & 0.5 \\
\hline BS60-1.5TMP-TA & 60 & 40 & 1.5 & 1 & 0.5 \\
\hline BS60-2TMP-TA & 60 & 40 & 2 & 1 & 0.5 \\
\hline BS60-1/2 PI & 60 & 40 & 1 & 0.5 & 0.25 \\
\hline BS60-1/4 PI & 60 & 40 & 1 & 0.25 & 0.125 \\
\hline
\end{tabular}

